\title{
Favorable Outcome of an Acute Complex Regional Pain Syndrome With Immunoglobulin Infusions
}

\author{
Friedrich Medlin, MD, Anastasia Zekeridou, MD, Susanne Renaud, MD, and Thierry Kuntzer, MD
}

Objective: To emphasize that complex regional pain syndrome (CRPS), a disabling disorder with the implication of aberrant inflammation, vasomotor dysfunction, and maladaptive neuroplasticity, might be treated with a high dose of intravenous immunoglobulin infusions (IVIG).

Methods: We describe a patient who presented with CRPS in the acute phase of the disease.

Results: The CRPS developed secondary to sciatic compression in a young patient and was treated within 10 days by high-dose IVIG $(2 \mathrm{~g} / \mathrm{kg})$. It resolved completely within days after infusions.

Discussion: This observational study emphasizes that high-dose IVIG may be a treatment option in the acute phase of CRPS.

Key Words: complex regional pain syndrome, IVIG, autoimmunity, neuropathic pain, autoantibodies

(Clin J Pain 2013;29:e33-e34)

omplex regional pain syndrome (CRPS) is a painful disorder characterized by a continuing regional pain with various combinations of abnormal sensory, motor, sudomotor, vasomotor, and trophic changes. ${ }^{1}$ The field has deviated from the traditional use of the CRPS-1/CRPS-2 dichotomy, underscoring the implication of aberrant inflammation, vasomotor dysfunction, and maladaptive neuroplasticity in the development of this disabling disorder. Even though the precise etiology of the CRPS is unknown, it is associated with an exaggerated inflammatory response with distinct profile characteristics between the acute and chronic form. ${ }^{2}$ According to the autoimmune hypothesis, with the identification of functionally active autoantibodies in certain CRPS patients, ${ }^{3}$ a recent randomized controlled trial of low-dose $(0.5 \mathrm{~g} / \mathrm{kg})$ intravenous infusions of human immunoglobulins (IVIG) showed significantly reduced pain intensity in the treated group among 13 chronic CRPS patients. ${ }^{4}$ We recently encountered a young disabled CRPS patient and treated him immediately with IVIG. The improvement was so significant that the case is briefly reported here, emphasizing that IVIG might also be a promising therapeutic alternative in the acute phase of the disorder.

Received for publication September 14, 2012; revised February 12, 2013; accepted March 10, 2013.

From the Nerve-Muscle Unit, Department of Clinical Neurosciences,

Lausanne University Hospital (CHUV) and University of Lausanne (UNIL), Lausanne, Switzerland.

The authors declare no conflict of interest.

Reprints: Thierry Kuntzer, MD, Service de neurologie, CHUV bh7, rue du Bugnon 47, 1011 Lausanne, Switzerland (e-mail: thierry.kuntzer (a) chuv.ch)

Copyright (C) 2013 by Lippincott Williams \& Wilkins

\section{CASE REPORT}

D.C. is a 19-year-old man who recovered from a 7-hour duration mandibular osteotomy with a right foot drop. Serum creatine kinase levels were elevated $(3700 \mathrm{U} / \mathrm{L}$, normal <200). Examination revealed a right MRC grade 4 muscle weakness of the extensors of the foot and toes, a right Achilles areflexia, as well as hypoesthesia of the whole right foot. Electrodiagnostic studies revealed a nonhomogenous motor and sensory axonal loss of the right sciatic nerve. Two weeks later, the patient reported hyperalgesia and neuropathic pain of the entire right leg with mechanical hypersensitivity, edema, and erythema of the feet (Fig. 1). In line with the "Budapest" criteria ${ }^{5}$ CRPS was diagnosed. As maximal dosage of pregabalin and tramadol did not reduce the intensity of the pain, infusions of IVIG (total dose of $2 \mathrm{~g} / \mathrm{kg}$ ) were given over 4 days. Within the first day of the IVIG treatment, pain decreased continuously and 2 weeks after the CRPS onset (1 mo after surgery), the pain and skin manifestations completely disappeared with a slight residual muscle weakness of the foot extensor. Soon after, he took up his employment and had no further functional restrictions.

\section{CONCLUSIONS}

The most likely diagnosis for the patient is postsurgical compressive sciatic neuropathy with secondary CRPS. The elevated creatine kinase levels and the long operation in reclined position in a slim patient make a postsurgical inflammatory neuropathy, in this context, unlikely. ${ }^{6}$ The role of the posttraumatic inflammation mediated by proinflammatory cytokines and neuropeptides

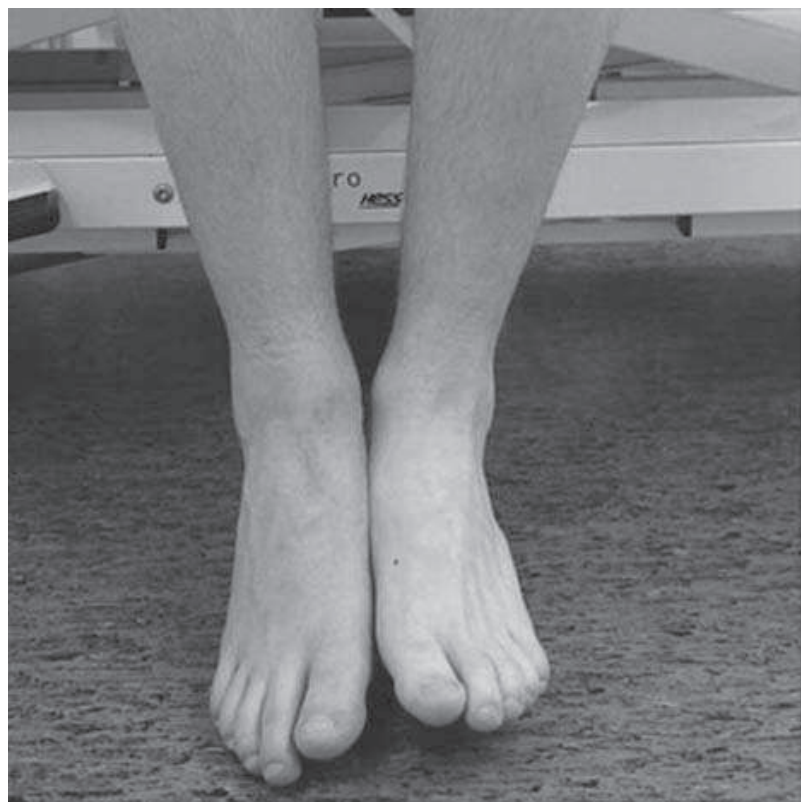

FIGURE 1. Image of the patient 2 weeks after the surgery, demonstrating features of acute CRPS of the right foot, with swelling and hyperemia. 
such as substance $\mathrm{P}$ is well documented in CRPS, ${ }^{1}$ and this pathway might be the target of the beneficial action of IVIG. It is believed that IVIG counteracts the ongoing neuroinflammation by multiple mechanisms such as Fc-receptor blockade, inhibition of complement deposition, neutralization of cytokines and growth factors, acceleration of autoantibodies clearance, as well as the activation of regulatory macrophages and $\mathrm{T}$ cells through the Fc $\gamma$ RIIb receptor. ${ }^{7,8}$ However, there is still limited evidence of IVIG treatment for CRPS and the timing, therapeutic doses, and duration of IVIG infusions are not yet known in CRPS.

Our reported patient underscores that early and high IVIG doses ( 2 vs. $0.5 \mathrm{~g} / \mathrm{kg}$ ) might speed the healing of this disabling disease in some patients. Further multicenter trials are necessary to determine who should receive IVIG.

\section{REFERENCES}

1. Marinus J, Moseley GL, Birklein F, et al. Clinical features and pathophysiology of complex regional pain syndrome. Lancet Neurol. 2011;10:637-648.
2. Parkitny L, McAuley JH, Di Pietro F, et al. Inflammation in complex regional pain syndrome. Neurology. 2013;80:106-117.

3. Kohr D, Singh P, Tschernatsch M, et al. Autoimmunity against the beta(2) adrenergic receptor and muscarinic-2 receptor in complex regional pain syndrome. Pain. 2011;152: 2690-2700.

4. Goebel A, Baranowski A, Maurer K, et al. Intravenous immunoglobulin treatment of the complex regional pain syndrome: a randomized trial. Ann Intern Med. 2010;152: $152-158$.

5. Harden RN, Bruehl S, Stanton-Hicks M, et al. Proposed new diagnostic criteria for complex regional pain syndrome. Pain Med. 2007;8:326-331.

6. Staff NP, Engelstad JN, Klein CJ, et al. Post-surgical inflammatory neuropathy. Brain. 2010;133:2866-2880.

7. Dirckx M, Stronks DL, Groeneweg G, et al. Effect of immunomodulating medications in complex regional pain syndrome. Clin J Pain. 2012;28:355-363.

8. Binkley K. Improving the diagnosis and treatment of CRPS: insights from a clinical immunologist's personal experience with an underrecognized neuroinflammatory disorder. $J$ Neuroimmune Pharmacol. 2012. [Epub ahead of print]. 